Case report

\title{
A Case Report of Laparoscopic Resection of a Large Leiomyoma of the Ovary That Required an Operation Time of Seven Hours
}

\author{
Satoru Takeuchi \\ Gynecology and Obstetrics, Kochi Health Sciences Center, Kochi, Japan
}

\begin{abstract}
Uterine leiomyoma is a common benign tumor. This report concerns a rare case of laparoscopic resection of a unilateral, ovarian leiomyoma requiring seven hours of surgery. A 66-yearold woman (para 2) was referred to Kochi Health Sciences Center because of a pelvic tumor. Transvaginal ultrasonographic examination revealed a pelvic tumor suspected of being a uterine leiomyoma in the cul-de-sac. Total laparoscopic hysterectomy and bilateral salpingo-oophorectomy was performed. A morcellator was used to reduce the volume of the leiomyoma. The uterus and right adnexa were removed via the vagina. When the left ovarian leiomyoma was diminished, it was also removed via the vagina.
\end{abstract}

Key words: leiomyoma of the ovary, laparoscopic surgery

(J Rural Med 2009; 4(1): 35-37)

\section{Introduction}

Uterine leiomyoma is a common benign tumor. Almost all leiomyomas grow from the uterus. Nonspecific benign mesenchymal tumors of the ovary are rare, but excepting pure fibromas, leiomyomas are the most frequent ${ }^{1)}$. Primary leiomyoma of the ovary is of unknown origin. It was originally described by Sangalli in $1862^{2}$.

We report a rare case of laparoscopic resection of a unilateral, ovarian leiomyoma requiring seven hours of surgery.

\section{Patient}

A 66-year-old woman (para 2) was referred to Kochi Health Sciences Center because of a pelvic tumor. Trans-

\section{Correspondence to: Satoru Takeuchi}

Gynecology and Obstetrics, Kochi Health Sciences Center, 2125-1 Ike, Kochi 781-8555, Japan

E-mail: satoru_takeuchi@khsc.or.jp vaginal ultrasonographic examination revealed a pelvic tumor with a maximum diameter of $11.8 \mathrm{~cm}$ suspected of being a subserous uterine leiomyoma in the cul-de-sac (Figure 1). CT showed a large pelvic tumor in the cul-de-sac (Figure 2). MRI indicated that the tumor was a leiomyoma (Figure 3). On magnetic resonance imaging, the tumor
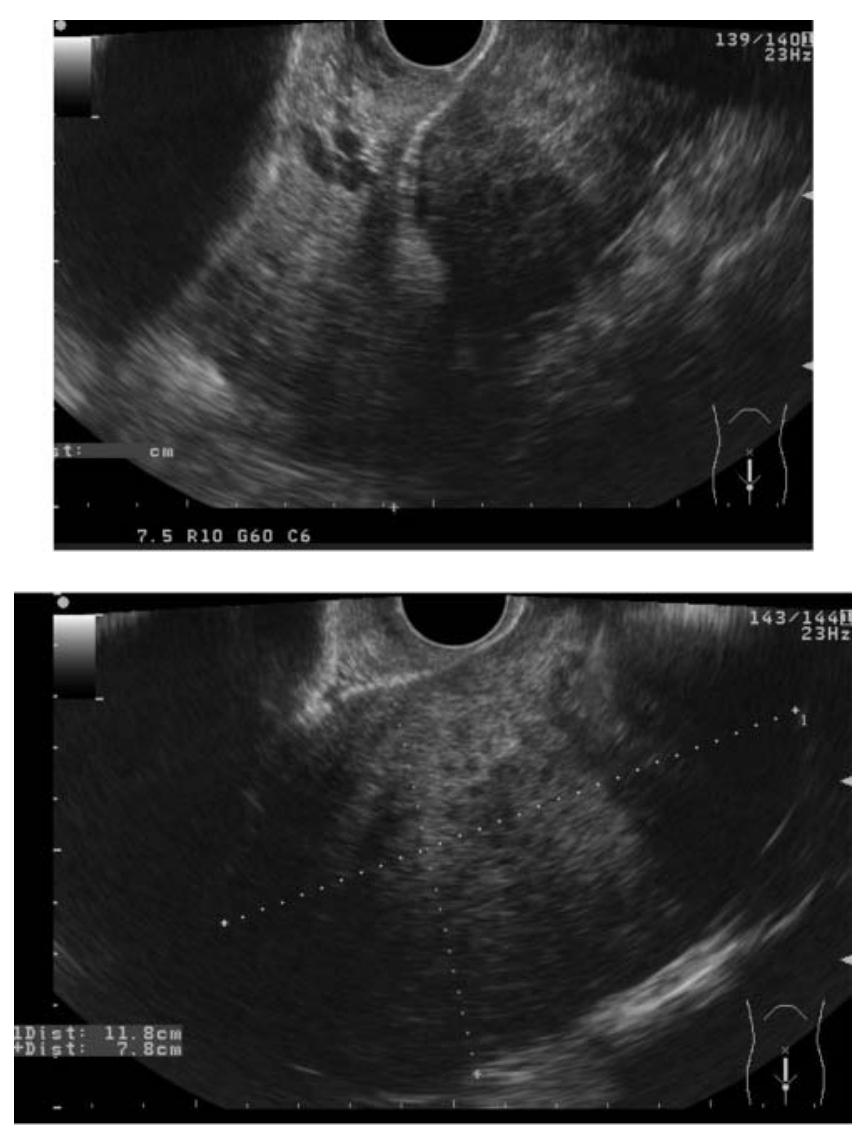

Figure 1 Transvaginal ultrasonography of the leiomyoma of the left ovary.

C2009 The Japanese Association of Rural Medicine 


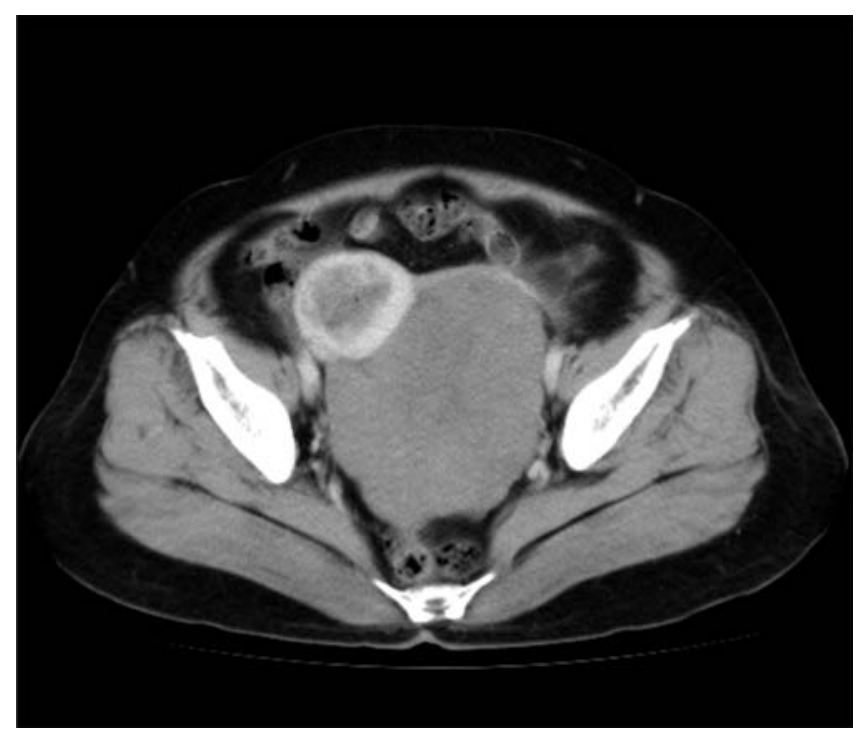

Figure 2 CT imaging of the leiomyoma of the left ovary.
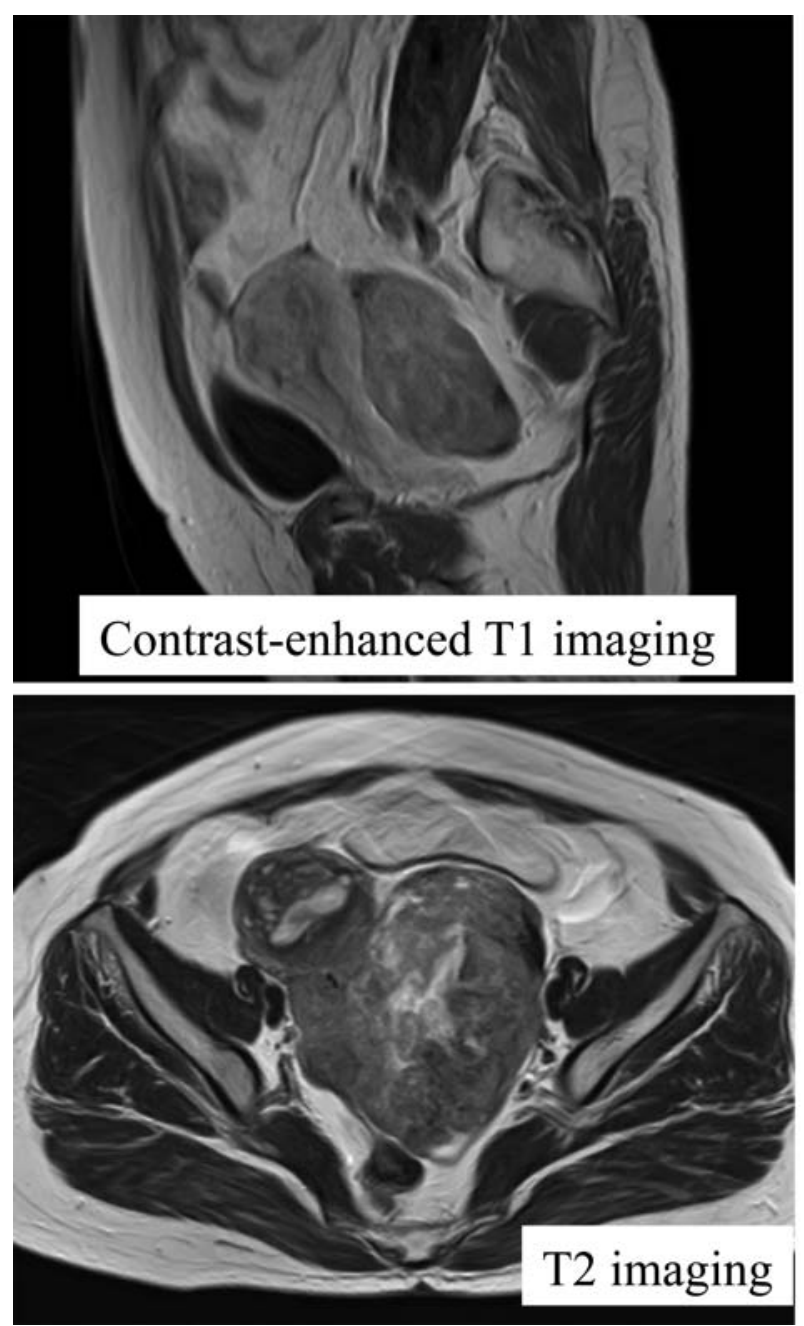

appeared as a well-circumscribed low signal intensity mass on T1 weighted images, with mixed signal intensity on T2 weighted imaging. Areas of high signal intensity on T2 weighted images corresponded to degeneration of the leiomyoma. Preoperative imaging studies indicated leiomyoma. We diagnosed the tumor as a subserous uterine leiomyoma. Total laparoscopic hysterectomy (TLH) and bilateral salpingo-oophorectomy were planned.

The patient underwent a laparoscopic surgery. Laparoscopic examination revealed that the tumor had grown from the left ovary and that the uterus was normal in size. The tumor was in the cul-de-sac and was hard to move to the anterior pelvic cavity. The bottom of the tumor was adhered to the surface of the rectum. The tumor was finally freed from the rectum, but the serosa of the rectum was torn. The surface of the rectum was subsequently sutured with 2-0 Vicryl. Bilateral salpingo-oophorectomy and hysterectomy were performed laparoscopically. The uterus and right adnexa were removed via the vagina. Volume reduction of the leiomyoma was performed with a morcellator. Macroscopi-

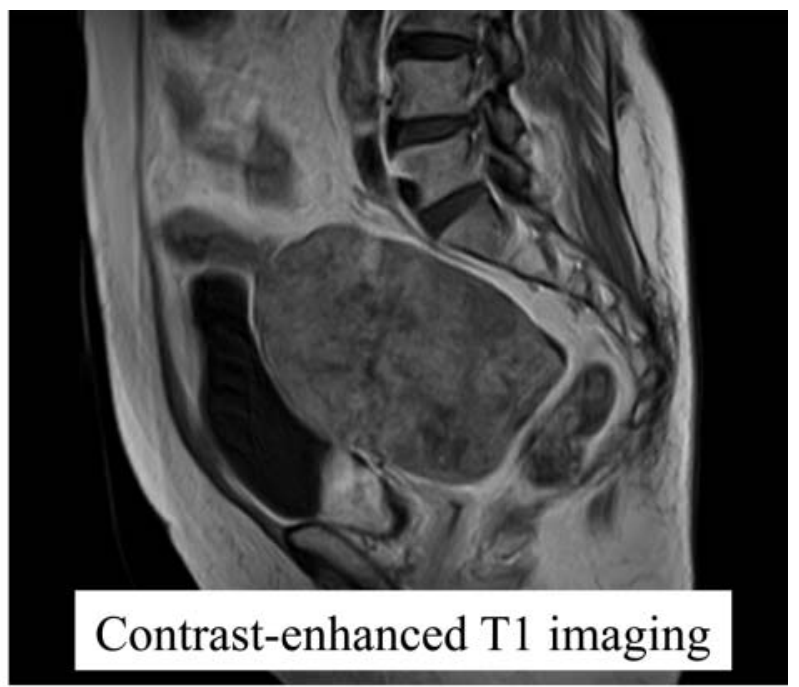

Figure 3 MRI imaging of the leiomyoma of the left ovary. 
cally, the cut surface of the tumor showed yellowish multilobular areas. There was no sign of necrosis or hemorrhage within the tumor. When the left ovarian leiomyoma was diminished, it was also removed via the vagina. The vaginal stump was closed with continuous 1-0 Vicryl. Hemostasis was achieved with a bipolar coagulator. The operating time was seven hours, and blood loss was $450 \mathrm{ml}$. The patient's postoperative course was uneventful. The results of histopathological examination indicated that the tumor was a benign leiomyoma of the left ovary. The patient was discharged on the sixth day after the operation.

\section{Discussion}

Leiomyomas are common benign tumors. The uterine corpus is the most common site of location. The origin and development of leiomyomas are not well understood, but they probably arise from smooth muscle cells ${ }^{3)}$. Several theories have been reported in regard to the histogenesis of these tumors. Some evidence suggests that they arise from the smooth muscle of blood vessel walls ${ }^{3)}$. Therefore, they can originate wherever smooth muscle cells exist ${ }^{3}$. The most readily acceptable explanation is that ovarian leiomyomas arise from the smooth muscle of the ovarian ligaments that enter the ovary or from the smooth muscle of ovarian blood vessels ${ }^{2}$. It is suspected that estrogen plays a role in the development and/or maintenance of uterine leiomyomas ${ }^{3)}$. Evidence of hormonal dependence includes increased frequency of leiomyomas after menarche, enlargement of leiomyomas during pregnancy and regression of leiomyomas after menopause ${ }^{3)}$. Because the ovary is theoretically the most dense organ of estrogen, leiomyomas can sometimes arise from the ovary. With few exceptions, ovarian leiomyomas are incidental findings of routine studies of the ovaries because they usually measure $<3 \mathrm{~cm}$ in diameter, rarely become large enough to present as a pelvic mass and are clinically indistinguishable from subserous leiomyomas and ovarian fibromas until surgery or histopathological confirmation $^{4}$. In the differential diagnosis, large subserous pedunculated leiomyoma or low cellular, primarily benign ovarian tumor are considered. In the present case, the operation time was lengthened by TLH and manipulation of a morcellator. If the ovarian leiomyoma had been diagnosed preoperatively, laparoscopic left adnexectomy might have been considered.

We found the ovarian myoma during laparoscopic surgery and resected it laparoscopically.

\section{References}

1. Matamala MF, Nogales FF, Aneiros J, et al. Leiomyoma of the ovary. In J Gynecol Pathology 1988; 7: 190-196.

2. Tsalacopoulos G, Tiltman AJ. Leiomyoma of the ovary. S Afr Med J 1981; 59: 574-575.

3. Dursun P, Salman MC, Taskiran C, et al. Retroperitoneal leiomyomatosis: a case report. Int J Gynecol Cancer 2005; 15: 1222-1225.

4. Roue A, Laboisse C, Winer N, et al. Extra-uterine pelvic leiomyoma: diagnosis and practical management. J Gynecol Obstet Biol Reprod (Paris) 2007; 36: 403-408. 